\title{
A NOTE ON HIGHER DERIVATIONS AND INTEGRAL DEPENDENCE
}

\author{
WILLIAM C. BROWN
}

\begin{abstract}
In this note we prove the following: Theorem. Let $R^{\prime}$ be an associative commutative ring with identity. Suppose $R^{\prime}$ is an integral extension of $R$, and $\delta=\left\{\delta_{i}\right\}$ is a higher derivation on $R^{\prime}$ which restricts to a higher derivation on $R$. Suppose $p$ is a prime ideal in $R$ which is differential under $\delta$. Then there exists a prime ideal $p^{\prime}$ in $R^{\prime}$ such that $p^{\prime}$ is $\delta$-differential and $p^{\prime} \cap R=p$.
\end{abstract}

Introduction. In this paper, we assume all rings are associative, commutative and have an identity. A subring of a given ring is assumed to have the same identity as the given ring.

Let $R$ be a ring. A higher derivation $\delta=\left\{\delta_{q}\right\}$ (of infinite rank) on $R$ is an infinite sequence of maps $\delta_{q}: R \rightarrow R, q=1,2,3, \cdots$, such that

(a) each $\delta_{q}$ is an additive group homomorphism;

(b) for all $x, y \in R$ and $q \geqq 1$,

$\delta_{q}(x y)=x \delta_{q}(y)+\delta_{1}(x) \delta_{q-1}(y)+\cdots+\delta_{q-1}(x) \delta_{1}(y)+\delta_{q}(x) y$

(Leibnitz's rule).

We shall abbreviate the last equation by writing $\delta_{q}(x y)=\sum_{i+j=q} \delta_{i}(x) \delta_{j}(y)$. We note that (a) and (b) imply that $\delta_{q}(1)=0$ for all $q$. Thus if $Z_{0}$ denotes the prime subring of $R$, i.e. the subring of $R$ generated by the identity element 1 , then $\delta_{q}\left(Z_{0}\right)=0$ for all $q \geqq 1$.

If $\delta$ is a higher derivation on $R$ and $A$ is an ideal in $R$, then we shall say $A$ is $\delta$-differential if $\delta_{q}(A) \subset A$ for all $q \geqq 1$.

The purpose of this paper is to prove the following:

THEOREM 1. Let $R^{\prime}$ be a ring containing $R$. Suppose $R^{\prime}$ is an integral extension of $R$, and $\delta$ is a higher derivation on $R^{\prime}$ which restricts to a higher derivation on $R$. Suppose $p$ is a prime ideal in $R$ which is differential under $\delta$. Then there exists a prime ideal $p^{\prime}$ in $R^{\prime}$ such that $p^{\prime}$ is $\delta$-differential and $p^{\prime} \cap R=p$.

This theorem appears as Theorem 2 in S. Sato's On rings with a higher derivation [2]. In his proof of this result, Sato seems to assume $R^{\prime}$ is

Received by the editors December 16, 1971.

AMS 1970 subject classifications. Primary 16A72.

Key words and phrases. Higher derivations, differential ideals.

(c) American Mathematical Society 1972 
Noetherian. If we assume $R^{\prime}$ is not Noetherian, then there is a gap in Sato's theorem which we shall fill in this note.

Proof of TheOrem 1. Following the first paragraph of Sato's proof, we can assume without loss of generality that $R$ is a quasi-local ring with maximal ideal $p$. Since $R^{\prime}$ is an integral extension of $R, p R^{\prime} \neq R^{\prime}$. Clearly $p R^{\prime}$ is a $\delta$-differential ideal in $R^{\prime}$. Hence by [2, Theorem 1] there exists a maximal $\delta$-differential ideal $p^{\prime}$ in $R^{\prime}$ which contains $p R^{\prime}$. If $p^{\prime}$ is a prime ideal, then the theorem is complete. Hence we wish to prove that $p^{\prime}$ is a prime ideal in $R^{\prime}$.

Consider $\left(R^{\prime}\right)=R^{\prime} / p^{\prime}$. Since $p^{\prime} \cap R=p,\left(R^{\prime}\right)^{-}$is an integral extension of the field $\bar{R}=R / p$. Since both $p$ and $p^{\prime}$ are $\delta$-differential ideals, $\delta$ induces a higher derivation $\bar{\delta}$ on $\left(R^{\prime}\right)^{-}$which restricts to a higher derivation on $\bar{R}$. Specifically, for all $q \geqq 1, \bar{\delta}_{q}\left(r+p^{\prime}\right)=\delta_{q}(r)+p^{\prime}$ for $r \in R^{\prime}$. Note that $\left(R^{\prime}\right)^{-}$has no proper $\bar{\delta}$-differential ideals. Otherwise $p^{\prime}$ would not be a maximal $\delta$-differential ideal in $R^{\prime}$.

Now let $N$ be any maximal ideal in $\left(R^{\prime}\right)^{-}$. If $N \neq 0$, then $\bar{\delta}(N) \notin N$. Hence there exists a nonzero element $x \in N$ such that $\bar{\delta}_{q}(x) \notin N$ for some $q \geqq 1$. Since $\left(R^{\prime}\right)^{-}$is an integral extension of $\bar{R}, x$ satisfies some monic polynomial $f(X) \in \bar{R}[X]$. Let $f(X)=X^{n}+r_{n-1} X^{n-1}+\cdots+r_{1} X+r_{0}$. Since $\bar{R}$ is a field, $f(x)=0$ implies $r_{0} \in N$. Hence $r_{0}=0$. So $f(X)=X^{n}+r_{n-1} X^{n-1}+$ $\cdots+r_{1} X$. We first argue that some $r_{i}, i=1, \cdots, n-1$, is not zero. That is, $x$ cannot be nilpotent. This follows from the following lemma:

LeMma 1. If $x^{n}=0$, then $\bar{\delta}_{q}(x) \in N$ for all $q \geqq 1$.

Proof. This lemma is argued via induction on $q$. Suppose $\bar{\delta}_{1}(x) \notin N$. Then we shall show that $n$ is bigger than every positive integer. Since $x \neq 0, n>1$. Since $\bar{\delta}_{2}\left(x^{n}\right)=0$, we get $\bar{\delta}_{1}\left(x^{n-1}\right) \in N$. Hence $n>2$. Assume we have shown $n>m \geqq 2$. Successively applying $\bar{\delta}_{2}, \cdots, \bar{\delta}_{m}$ to the equation $x^{n}=0$, we get $\bar{\delta}_{1}\left(x^{n-1}\right) \in N, \bar{\delta}_{2}\left(x^{n-1}\right)$ and $\bar{\delta}_{1}\left(x^{n-2}\right) \in N, \cdots, \bar{\delta}_{m-1}\left(x^{n-1}\right)$ through $\bar{\delta}_{1}\left(x^{n-(m-1)}\right) \in N$. Now

$$
0=\bar{\delta}_{m+1}\left(x^{n}\right)=\sum_{i+j=m+1} \bar{\delta}_{i}(x) \bar{\delta}_{j}\left(x^{n-1}\right) .
$$

Thus, $\quad \bar{\delta}_{m}\left(x^{n-1}\right) \in N$. But $\bar{\delta}_{m}\left(x^{n-1}\right)=\sum_{i+j=m} \bar{\delta}_{i}(x) \bar{\delta}_{j}\left(x^{n-2}\right)$. Therefore $\bar{\delta}_{m-1}\left(x^{n-2}\right) \in N$. By expanding this further, we get $\bar{\delta}_{1}\left(x^{n-m}\right) \in N$. Hence $n>m+1$. Thus $\bar{\delta}_{1}(x) \in N$.

Assume we have shown that $\bar{\delta}_{1}(x), \cdots, \bar{\delta}_{q-1}(x) \in N$. If $\bar{\delta}_{q}(x) \notin N$, we shall again show that $n$ is bigger than every positive integer. The procedure is similar to the case $q=1$. Applying $\bar{\delta}_{2 q}$ to the equation $x^{n}=0$ we get $\bar{\delta}_{q}\left(x^{n-1}\right) \in N$. So $n>2$. Applying $\bar{\delta}_{2 q+1}, \cdots, \bar{\delta}_{3 q}$ to $x^{n}=0$, we get $\bar{\delta}_{q+1}\left(x^{n-1}\right), \cdots, \bar{\delta}_{2 q}\left(x^{n-1}\right)$ are all in $N$. But

$$
\bar{\delta}_{2 q}\left(x^{n-1}\right)=\sum_{i+j=2 q} \bar{\delta}_{i}(x) \bar{\delta}_{j}\left(x^{n-2}\right)
$$


being an element of $N$ implies $\bar{\delta}_{q}\left(x^{n-2}\right) \in N$. Thus $n>3$. I think it is clear now how one proceeds by induction to show $n>m$ for any $m$. Thus $\delta_{q}(x) \in N$, and the proof of Lemma 1 is complete.

Lemma 1 implies that $x$ cannot be nilpotent; for $x$ was chosen in $N$ such that $\bar{\delta}_{q}(x) \notin N$ for some $q$. Thus some $r_{i}$ in $f(X)$ must be nonzero. We shall now show that this implies $\bar{\delta}_{q}(x) \in N$ for all $q \geqq 1$. Thus $N=0$ and therefore $\left(R^{\prime}\right)^{-}$is a field. This implies that $p^{\prime}$ is a maximal ideal and completes the proof of the theorem.

We shall show that $\bar{\delta}_{q}(x) \in N$ for all $q$ by induction on $q$.

Suppose $\bar{\delta}_{1}(x) \notin N$. We need the following lemma:

LEMMA 2. If $\bar{\delta}_{1}(x) \notin N$, then $\bar{\delta}_{k}\left(x^{l}\right) \in N$ if $l>k$, and $\bar{\delta}_{k}\left(x^{l}\right) \notin N$ if $l=k$.

Proof. We prove this lemma via induction on $k$. If $k=1$, the result follows easily from the hypothesis and Leibnitz's rule. So assume the lemma holds if $k=1, \cdots, m$. Then

$$
\bar{\delta}_{m+1}\left(x^{m+1}\right)=\sum_{i+j=m+1} \bar{\delta}_{i}(x) \bar{\delta}_{j}\left(x^{m}\right)
$$

which in turn is congruent modulo $N$ to $\bar{\delta}_{1}(x) \bar{\delta}_{m}\left(x^{m}\right)$. But $\bar{\delta}_{1}(x) \bar{\delta}_{m}\left(x^{m}\right) \notin N$, therefore $\bar{\delta}_{m+1}\left(x^{m+1}\right) \notin N$.

If $l>1$, then $\bar{\delta}_{m+1}\left(x^{m+l}\right) \in N$ follows from repeated applications of Leibnitz's rule.

Using Lemma 2, we can now show $\bar{\delta}_{1}(x) \notin N$ implies every $r_{i}, i=1, \cdots$, $n-1$, in $f(X)$ is zero. Applying $\bar{\delta}_{1}$ to the equation $f(x)=0$, we get

$$
0=\bar{\delta}_{1}(f(x))=f^{\prime}(x) \bar{\delta}_{1}(x)+\bar{\delta}_{1}\left(r_{n-1}\right) x^{n-1}+\cdots+\bar{\delta}_{1}\left(r_{1}\right) x
$$

where $f^{\prime}(X)$ is the formal derivative of $f(X)$. Since $\bar{\delta}_{1}(x) \notin N, f^{\prime}(x) \in N$. Therefore $r_{1}=0$. So

$$
f(X)=X^{n}+r_{n-1} X^{n-1}+\cdots+r_{2} X^{2}
$$

If we apply $\bar{\delta}_{2}$ to $f(x)=0$, we get

$$
0=\bar{\delta}_{2}\left(x^{n}\right)+\sum_{i+j=2} \bar{\delta}_{i}\left(r_{n-1}\right) \bar{\delta}_{j}\left(x^{n-1}\right)+\cdots+\sum_{i+j=2} \bar{\delta}_{i}\left(r_{2}\right) \bar{\delta}_{j}\left(x^{2}\right) .
$$

Applying Lemma 2, we get $r_{2} \bar{\delta}_{2}\left(x^{2}\right) \in N$. But $\bar{\delta}_{2}\left(x^{2}\right) \notin N$. Thus $r_{2}=0$. By applying $\bar{\delta}_{3}, \cdots, \bar{\delta}_{n-1}$ to $f(x)=0$ and applying Lemma 2 , we get $r_{n-1}=\cdots=r_{3}=0$. This is a contradiction, and hence $\bar{\delta}_{1}(x) \in N$. So assume we have shown that $\bar{\delta}_{1}(x), \cdots, \bar{\delta}_{m-1}(x) \in N(m>1)$. Assume $\bar{\delta}_{m}(x) \notin N$. Again we shall show that this leads to $r_{n-1}=\cdots=r_{1}=0$. We need:

LeMma 3. $\quad \bar{\delta}_{k h_{l}}\left(x^{l}\right) \in N$ if $l>k$, and $\bar{\delta}_{k m}\left(x^{l}\right) \notin N$ if $l=k$. 
Proof. We prove this lemma via inductinn on $k$. If $k=1$, then $\bar{\delta}_{m}(x) \notin N$ by hypothesis. $\left.\bar{\delta}_{m}\left(x^{2}\right)=\sum_{i+j=m} \bar{\delta}_{i}(x), \bar{s}_{j}, x\right) \in N$ since $\bar{\delta}_{1}(x), \cdots$, $\bar{\delta}_{m-1}(x) \in N$. If $\bar{\delta}_{m}\left(x^{l}\right) \in N$ for $l=2, \cdots, t$, then $\ddot{\theta}_{m}\left(x^{l+t}\right) \in N$ by an easy application of Leibnitz's rule. Hence the lemma holds for $k=1$.

Assume the result holds for $k=1, \cdots, r$. Applying Leibnitz's rule again, we get

$$
\bar{\delta}_{(r+1) m}\left(x^{r+1}\right)=\sum_{i_{1}+\cdots+i_{r+1}=(r+1) m} \bar{\delta}_{i_{1}}(x) \cdots \bar{\delta}_{i_{r+1}}(x) .
$$

This sum is clearly congruent modulo $N$ to $\bar{\delta}_{m}(x)^{r+1}$ which is not an element of $N$. Thus $\bar{\delta}_{(r+1) m}\left(x^{r+1}\right) \notin N$. A similar argument shows

$$
\bar{\delta}_{(r+1) m}\left(x^{r+l}\right) \in N \text { if } l>1 \text {. }
$$

If we now apply $\bar{\delta}_{m}$ to $f(x)=0$, we get

$$
0=\bar{\delta}_{m}(f(x))=\bar{\delta}_{m}\left(x^{n}\right)+\sum_{i+j=m} \bar{\delta}_{i}\left(r_{n-1}\right) \bar{\delta}_{j}\left(x^{n-1}\right)+\cdots+\sum_{i+j=m} \bar{\delta}_{i}\left(r_{1}\right) \bar{\delta}_{j}(x)
$$

Applying Lemma 3 and the induction hypothesis, we get $r_{1} \bar{\delta}_{m}(x) \in N$. Hence $r_{1}=0$. Successively applying $\bar{\delta}_{2 m}, \cdots, \bar{\delta}_{(n-1) m}$ to $f(x)=0$, we get $r_{2}=\cdots=r_{n-1}=0$. Thus every $r_{i}$ in $f(X)$ is zero. This is a contradiction. Hence $\bar{\delta}_{q}(x) \in N$ for all $q$. Hence the theorem is completely proven.

COROllaRy 1. Let $R^{\prime}$ be an integral extension of $R$, and let $R$ contain a field of characteristic zero. Let $\delta$ be an ordinary derivation of rank one on $R^{\prime}$ such that $\delta$ restricts to a derivation on $R$. If $p$ is a $\delta$-differential prime ideal of $R$, then there exists a $\delta$-differential prime ideal $p^{\prime}$ in $R^{\prime}$ such that $p^{\prime} \cap R=p$.

Proof. The corollary follows immediately from Theorem 1 since $\delta$ can be embedded as the first term in the higher derivation $\delta^{\prime}=\left\{\delta^{i} / i\right.$ ! $\}$ on $R^{\prime}$.

We note that Corollary 1 is false in the characteristic $r \neq 0$ case. Consider the following simple example:

Let $Z_{5}$ denote the integers modulo 5 . Let $X$ denote an indeterminate over $Z_{5}$ and consider $R^{\prime}=Z_{5}[X] /\left(X^{5}\right)=Z_{5}[x]$. Then $R^{\prime}$ is a local ring with maximal ideal generated by $x$. Clearly $R^{\prime}$ is an integral extension of $Z_{5}$. Let $\delta$ be a derivation of rank one on $Z_{5}[X]$ defined by $\delta(X)=1$. Then $\delta\left(X^{5}\right) \subset\left(X^{5}\right)$. Hence, $\delta$ induces a derivation $\bar{\delta}: R^{\prime} \rightarrow R^{\prime}$ which restricts to a derivation on $Z_{5}$. Now (0) is a $\bar{\delta}$-differential prime ideal in $Z_{5}$, but there is no $\bar{\delta}$-differential prime ideal in $R^{\prime}$ which lies over it. This example also shows us that the associated prime ideals of a differential ideal need not in general be differential. (This result is true in the characteristic zero case [3, Theorem 1].) 
Using Theorem 1 we can easily prove the going up theorem for differential prime ideals.

COROLlary 2. With the same hypotheses as in Theorem 1, suppose $p_{1} \subset p_{2} \subset \cdots \subset p_{n}$ is a chain of $\delta$-differential prime ideals in $R$. Then there exists a chain of $\delta$-differential prime ideals $p_{1}^{\prime} \subset \cdots \subset p_{n}^{\prime}$ in $R^{\prime}$ such that $p_{i}^{\prime} \cap R=p_{i}$.

Proof. By Theorem 1 , there exists a $\delta$-differential prime ideal $p_{1}^{\prime}$ in $R^{\prime}$ lying over $p_{1}$. Passing to the residue class rings $R^{\prime} / p_{1}^{\prime}$ and $R / p_{1}$ and applying Theorem 1 again, we get a $\bar{\delta}$-differential prime ideal $q$ in $R^{\prime} \mid p_{1}^{\prime}$ which lies over $p_{2} / p_{1}$. We can now pull $q$ back to a $\delta$-differential prime ideal $p_{2}^{\prime}$ which contains $p_{1}^{\prime}$ and lies over $p_{2}$. Continuing in this fashion, we construct the entire chain.

If we assume $R^{\prime}$ is Noetherian, we can prove the going down theorem also.

THEOREM 2. Suppose $R^{\prime}$ is a Noetherian integral extension of $R$. Assume $R$ is a normal ring in which no nonzero element is a zero divisor in $R^{\prime}$. Let $\delta$ be a higher derivation on $R^{\prime}$ which restricts to a higher derivation on $R$. Let $p \subset q$ be a chain of $\delta$-differential prime ideals in $R$, and let $q^{\prime}$ be $a$ $\delta$-differential prime ideal in $R^{\prime}$ lying over $q$. Then there exists a $\delta$-differential prime ideal $p^{\prime} \subset q^{\prime}$ such that $p^{\prime} \cap R=p$.

Proof. Consider the $\delta$-differential ideal $p R^{\prime}$. It is well known $[4$, p. 263] that any isolated prime of $p R^{\prime}$ contracts to $p$ in $R$. Further, by [2, Proposition 1] or for a detailed proof [1, Theorem 1], any isolated prime of $p R^{\prime}$ is $\delta$-differential. Since $p R^{\prime} \subset q^{\prime}, q^{\prime}$ must contain some isolated prime $p^{\prime}$ of $p R^{\prime}$. This is the required $\delta$-differential prime ideal lying over $p$.

\section{REFERENCES}

1. W. C. Brown and W. E. Kuan, Ideals and higher derivations in commutative rings, Canad. J. Math. (to appear).

2. S. Sato, On rings with a higher derivation, Proc. Amer. Math. Soc. 30 (1971), 63-69.

3. A. Seidenberg, Differential ideals in rings of finitely generated type, Amer. $\mathrm{J}$. Math. 89 (1967), 22-42. MR 35 \#2902.

4. O. Zariski and P. Samuel, Commutative algebra. Vol. 1, University Series in Higher Math., Van Nostrand, Princeton, N.J., 1958. MR 19, 833.

Department of Mathematics, Michigan State University, East lansing, MiCHIGAN 48823 